\title{
Recommended rehabilitation for trunk exercise with the concept of frontal, sagittal and horizontal plane
}

\begin{abstract}
From anatomical and physiological points of view, human perform various movements, which are combined with behaviors of trunk and extremities. In human body, proprioception system has been present between sensory receptors and central nervous system (CNS). For this mechanism, minute motions are possible. Trunk has to be trained with the concept with three dimensions, which are frontal, sagittal and horizontal planes. Pole exercise has been developed for rehabilitation in the coordination of the movements of trunk and extremities, including bend and extend, side bend, turn, slide, spiral and rotate.
\end{abstract}

Keywords: three dimensions, frontal, sagittal and horizontal planes, proprioception, pole exercise
Volume 5 Issue 4 - 2020

\author{
Akito Moriyasu, ${ }^{1,2}$ Mitsuru Murakami, ${ }^{3}$ \\ Hiroshi Bando ${ }^{4,5}$ \\ 'Rehabilitation Research Group for body and heart in Shikoku, \\ Japan \\ ${ }^{2}$ Akiboshi Bright Star training rehabilitation center, Japan \\ ${ }^{3}$ Japan Masters Athletics, Kagawa division, vice-president, Japan \\ ${ }^{4}$ Medical Research, Tokushima University, Japan \\ 5 Japan Masters Athletics, Tokushima division, board, Japan
}

Correspondence: Hiroshi Bando, Medical Research, Tokushima University, Japan Masters Athletics, Tokushima division, board, Japan, Nakashowa I-61, Tokushima 770-0943,

Tel +8I-90-3|87-2485, Email pianomed@bronze.ocn.ne.jp

Received: July 25, 2020 | Published: August 14, 2020

\section{Introduction}

From anatomical and physiological points of view, human perform various movements, which are combined with behaviors of trunk and extremities. It is due to fundamental trunk mechanism that the extremities can move freely. Our trunk generally consists of three parts, thorax, vertebrae and pelvis. ${ }^{1}$ In addition, various joints such as facet joints, costal joints, and sacroiliac joints exist and are involved in all kinds of movements. ${ }^{2}$

We can usually move smoothly as we like. It is possible because the central nervous system (CNS) can collect and integrate various information from sensory receptors and output the commands to the effectors. ${ }^{3}$ In this situation, accurate sensory input is indispensable to perform fine and highly accurate behavior. As a matter of fact, a large number of sensory receptors are distributed in the muscles, tendons, ligaments, and joint capsules. They make up the joints, and constantly send minute positional information on each joint to the CNS.

When there is any impaired function of joint, inaccurate sensory information will be sent to the CNS. Then, the inadequate information will result in incorrect movements. Consequently, by optimizing each joint function for the trunk with many joints, it is possible to perform highly accurate movements.

In other words, these mechanisms are recognized as proprioception system. ${ }^{4}$ They include 3 levels of motor control, which are spinal reflexes, cognitive programming, and brainstem activity. A series of proprioception function can be always related to neuromuscular control and joint stability. ${ }^{5}$ Rehabilitation continues to evolve with the increased emphasis on patient management and proprioceptive training. ${ }^{6}$

In general, exercise therapy has been widely known to everyone. The contents can be roughly divided into training for the trunk and for the extremities. However, they usually overlap each other and the core training would be more important. ${ }^{7}$ Exercise therapies for the core include stretch poles, inner muscle training and self-weight training.
What kind of functions are necessary to effectively improve the ability of the trunk? A variety of joints and muscles in the spine, rib cage and pelvis are always involved in all movements. ${ }^{8}$ Among them, to maintain functional connections has been required simultaneously, instead of moving them separately.

It is necessary to send appropriate sensory information to the receptor for the natural movements. In order to make this successful, one has to recognize three-dimension concepts in the joints and muscles of the trunk, rather than in 1-2 dimensions. ${ }^{9}$ Then, any movement becomes smooth without extra tension or waste of power. Consequently, this process and direction can give athletes higher level of performance.

All athletes want to move the trunk naturally and smoothly without unnecessary tension. In order to make it easier, to consider movement in the light of three dimensions would be recommended. ${ }^{10}$ There are three planes to recognize in the sports and rehabilitation (Figure 1). Authors and collaborators have often explained the phenomena in the following. They are

i. Frontal plane: axis of special movement that causes reptiles to make torsion and move forward (Figure 1a),

ii. Sagittal plane: axis of movement that is observed when rabbits jump forward in mammals (Figure 1b),

iii. Horizontal plane: axis of movement in which the monkey twists the pelvis and pushes half of the body forward (Figure 1c). These examples are useful to understand.

As to the red and green arrows in Fig.1a, these indicate the changes in body axis during moving forward. The sprawling posture shows the fundamental design principles and the constraints on 3-dimension kinematics of the body and limbs axis as its locomotion. ${ }^{11}$ Generally, locomotion of tetrapods involves coordinated efforts for appendicular and axial musculoskeletal systems. The interaction of limbs and the ground may generate horizontal, vertical and mediolateral groundreaction forces. ${ }^{12}$ 

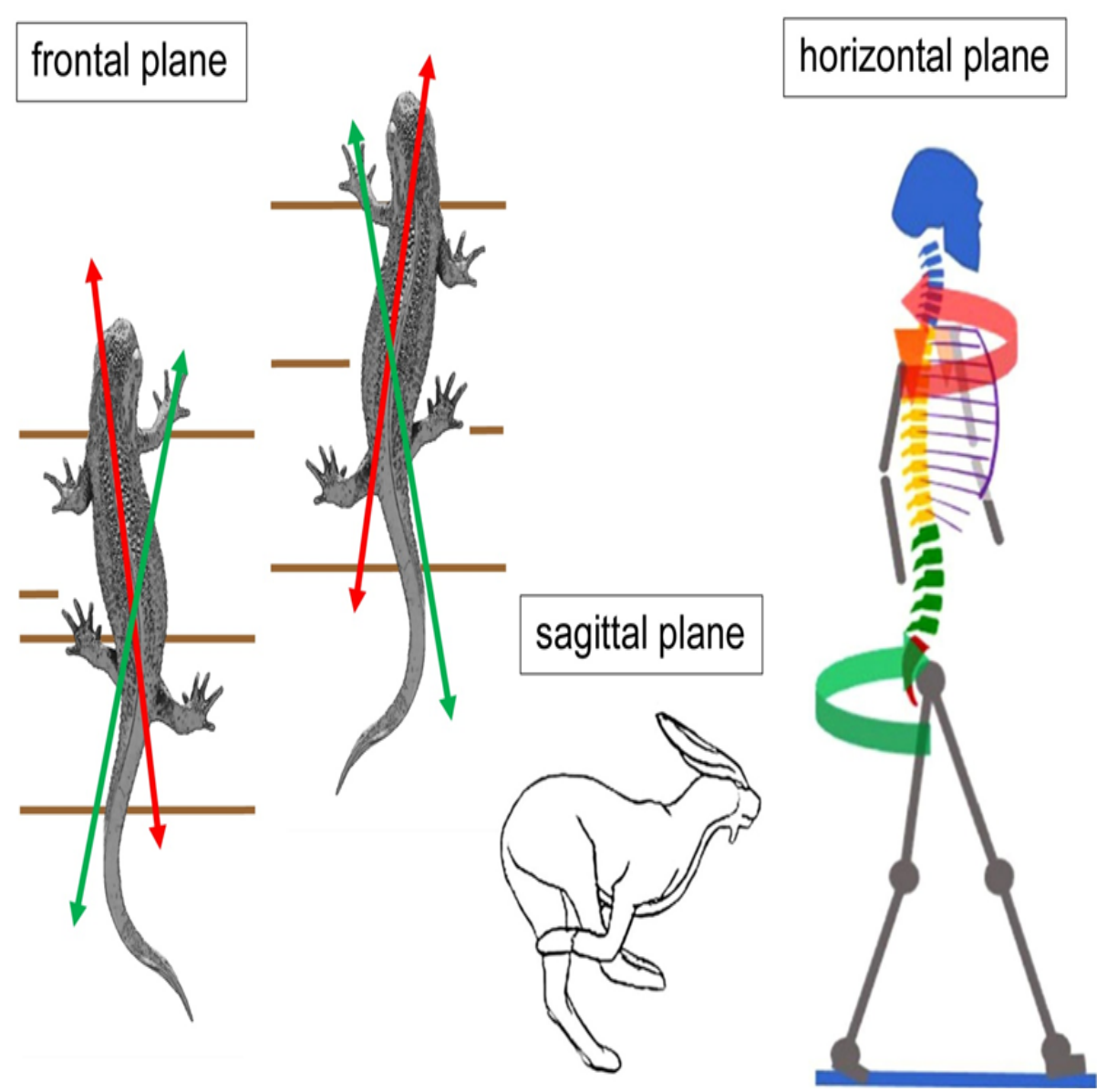

Figure I Axis of human movement in three-dimensional plane.

la: frontal plane

Ib: sagittal plane

Ic: horizontal plane.

As described above, we have to recognize a variety of movements in the trunk from the viewpoint of three dimensions. Human trunk is the core of the body, and it consists of mainly three components, which are the thorax, the spine and the pelvis. ${ }^{1}$ The thorax has a large degree for range of motion (ROM), because it can be twisted in a three-dimensional space. On the other hand, the latter two, spine and pelvis are rather restricted in their movements. ${ }^{10}$

From these situations, authors have so far developed pole exercises for a variety of subjects. As regards to the movement perspectives, pole exercise includes following 6 types of motion. They are bend and extend, side bend, turn, slide, spiral and rotate. ${ }^{13}$ By performing all these movements, three-dimensional exercise can be promoted, and stretching to the muscles can be also performed at the same time. Taking most advantages of these motions, orthopedic research has been reported using pole exercise. ${ }^{14}$ Successively, authors have spread research for various subjects including elderly people, patients with orthopedic diseases, professional athletes for baseball and football, Masters' athletes, junior and high school athletes, and so on. ${ }^{15}$

In summary, movements in humans progress from the core to the extremities. It is important to consider the movement of the trunk in three dimensions: frontal, sagittal, and horizontal. The authors have trained rehabilitating these movements with pole exercises and will continue enlightening them. This article becomes hopefully some reference in the future clinical research.

\section{Acknowledgments}

Authors express our gratitude for related people concerning this study.

\section{Conflicts of interest}

The authors declare no conflict of interest.

\section{References}

1. Narimani M, Arjmand N. Three-dimensional primary and coupled range of motions and movement coordination of the pelvis, lumbar and thoracic spine in standing posture using inertial tracking device. Journal of Biomechanics. 2018;69:169-174.

2. Manchikanti L, Schultz DM, Benyamin RM, et al. Thoracic Facet Joint Interventions. In: Manchikanti L, Kaye A, editors. Essentials of interventional techniques in managing chronic pain. Springer, Cham. 2018.

3. Marichal-Cancino A, Fajardo-Valdez B, Ruiz-Contreras AE, et al. Advances in the physiology of GPR55 in the central nervous system. Current Neuropharmacology. 2017;15(5):771-778. 
4. Lephart SM, Pincivero DM, Giraido JL, et al. The role of proprioception in the management and rehabilitation of athletic injuries. The American Journal of Sports Medicine. 1997;25(1):130-137.

5. Hagert E. Proprioception of the wrist joint: a review of current concepts and possible implications on the rehabilitation of the wrist. Journal of Hand Therapy. 2010;23(1):2-17.

6. Lee S, Kim H, Ye D, et al. Comparison of proprioception between osteoarthritic and age-matched unaffected knees: a systematic review and meta-analysis. Arch Orthop Trauma Surg. 2020.

7. Moriyasu A, Bando H, Akayama R, et al. Thorax flexibility can be increased by standing pole exercise. Int J Phys Med Rehabil. 2017;6:444.

8. Murakami M, Bando H, Moriyasu A. The concept of trunk connection can be applied for the training of short distance sprint. J Sports Med Rehabil. 2020;1(1):001

9. De Baets L, Van Deun S, Monari D, et al. Three-dimensional kinematics of the scapula and trunk, and associated scapular muscle timing in individuals with stroke. Human Movement Science. 2016;48:82-90.

10. Ebrahimi S, Kamali F, Razeghi M, et al. Comparison of the trunk-pelvis and lower extremities sagittal plane inter-segmental coordination and variability during walking in persons with and without chronic low back pain. Human Movement Science. 2017;52:55-66.
11. Russell A, Bels V. Biomechanics and kinematics of limb-based locomotion in lizards: review, synthesis and prospectus. Comparative Biochemistry and Physiology Part A: Molecular \& Integrative Physiology. 2001;131(1):89-112.

12. Capano JG. Reaction forces and rib function during locomotion in snakes. Integrative and Comparative Biology. 2020;60(1) 215-231.

13. Moriyasu A, Bando H, Murakami M. Pole exercise would be clinically effective through increasing thoracic spinal mobility. J Phys Med Rehabil. 2020;2019;1(1):03.

14. Kurihara R, Fujimoto D, Dakashita T, et al. The influence of pole exercise on the range of motion of thoracic spine. Clinical Research in Orthopaedics. 2019;2(1):1-5.

15. Bando H, Murakami M, Moriyasu A. Development of health and medical research for long similar to successful marathon running. J Health Med Res. 2019;1(1):3. 